\title{
Frontal Sinus Surgery Anterior to the Ethmoid Bulla
}

\author{
Junfeng Ji, Mei Zhou, Zeqing Li, Tianyou Wang, You Cheng, Qiuping Wang \\ Department of Otolaryngology and Head Neck Surgery, Jinling Hospital, Nanjing, China
}

\begin{abstract}
The frontal sinus surgery is difficult to perform but the ethmoid bulla is a relative, constant landmark in the middle turbinate that can improve the surgery. The purpose of this study was to evaluate the validity, security, and predominance of approaches to the frontal sinus via the route anterior to the ethmoid bulla. The data from 370 endoscopic frontal sinus surgery cases from our center were integrated and retrospectively analyzed. Three hundred twenty-nine patients underwent frontal sinus surgery via the route anterior to the ethmoid bulla. An additional 27 patients underwent frontal sinus surgery with mini-trephination, 13 patients with the Draf II procedure, and 1 patient had applied MELP (modified endoscopic Lothrop procedure). No serious complications occurred; however, there were 3 cases of eyelid ecchymosis and 1 case of anterior ethmoid artery bleeding. In all, 319 patients $(86.2 \%)$ were cured, an improvement was noted in 36 of the patients $(9.7 \%)$, and there was no improvement in 15 patients $(4.1 \%)$. Frontal sinus surgery via the route anterior to the ethmoid bulla is valid, relatively safe, and can be applied in most cases involving frontal disease.
\end{abstract}

Key words: Frontal sinus surgery - Ethmoid bulla - Endoscope

$\mathrm{T}$ he location of the frontal sinus is unique. There are many cells in the frontal recess that are pneumatized to various degrees. Additionally, the space in the frontal recess is narrow, so frontal sinusotomy is difficult. ${ }^{1}$ Endoscopic frontal sinusotomy has become the main procedure used to treat frontal sinus disease, yet two main challenges still exist. One involves the exact opening of the frontal sinus ostia, and the other is the overall safety of the procedure. Therefore, we applied the procedure of endoscopic frontal sinusotomy via the route anterior to the ethmoid bulla and found this approach to be technically simple, effective, and safe for treating the frontal sinus.

\section{Methods and Materials}

The procedures were performed by all authors. Five hundred forty-six patients who underwent endoscopic sinus surgery involving frontal sinus disease,

Reprint requests: Qiuping Wang, MD, Department of Otolaryngology and Head Neck Surgery, Jinling Hospital, 305 East Zhongshan Road, Nanjing, Jiangsu province, 210002, China.

Tel. and Fax: +86 25 80860106; E-mail: qpwang1016@126.com, papersubmit_mj@yahoo.com.cn 
in Jinling hospital between 2004 and 2009, were retrospectively analyzed. The main criteria that had been used to identify candidates on whom to perform frontal recess dissection were persistent symptoms of nasal blockage or congestion, headache, postnasal drip, and nasal discharge after antibiotic therapy for at least 4 weeks. The data from 370 patients were integrated and all completed the follow-up review. Of these, 245 patients were male and 125 were female; the overall case group had an average age of 37 years (range, 18-66 years). All patients underwent high-resolution computed tomography (CT) scans, endoscopic examinations of nasal cavity, and allergy examinations. The followup time exceeded at least three months, with a mean follow-up of 16.5 months (range, 6-46 months).

Various frontal sinus surgical methods were carried out depending on the extent, degree, and characteristics of the disease and the degree of difficulty of the surgery. These surgical methods included endoscopic frontal sinus surgery via the route anterior to the ethmoid bulla and endoscopic frontal sinus surgery with the help of mini-trephination, Draf II procedure, and MELP (modified endoscopic Lothrop procedure).

The methods, complications, and outcomes were collected and examined for potential statistically significant relationships. The optical system and rigid endoscopes used in all procedures were from the Karl Storz Company (Tuttlingen, Germany). In most cases, 30-degree rigid endoscopes were used; however, when the affected tissue was not clearly visible within their scope, 70-degree rigid endoscopes were used. The various forceps, microdebriders, and mini-trephines were manufactured by Medtronic-Xomed (Jacksonville, Florida).

The surgical technique (endoscopic frontal sinus surgery via the route anterior to the ethmoid bulla; Fig. 1) was initiated by first outlining the middle meatus. The middle meatus field was cleared of polyps, edema, and polypoid tissue to ensure an unobstructed view of the uncinate process, ethmoid bulla, middle turbinate, and hiatus semilunar. Before proceeding, the position of the uncinate process, ethmoid bulla, and middle turbinate, particularly the upper portions, were confirmed. The lower two thirds of the uncinate process was expected to be visible under this condition.

Next, the resection of the lower two thirds of the uncinate process was carried out. It was important to explore the exact border of the uncinate process and entirely resect it. The resection was carefully performed to avoid damaging the ethmoid bulla and the lamina papyracea. The endoscope was then placed into the middle meatus to observe the upper one third of the uncinate process and its relationship to the ethmoid bulla and middle turbinate. The anterior portion of the uncinate process and the anterosuperior portion of the middle turbinate combine to form the dome of the anterosuperior portion of middle meatus. The basal lamella of the uncinate process and the ethmoid bulla are connected in a perpendicular relationship. Kim identified the structure of this connection as the suprainfundibular plate (SIP). ${ }^{2}$ The cleft between the basal lamella of the uncinate process and the middle turbinate is known as the groove of the meatus.

The next step was to explore the ostia of the frontal sinus. After resection of the connection of the anterior portion of the uncinate process and the anterosuperior portion of the middle turbinate, the dome-like entrance of the middle meatus can be established. The middle meatus can be widely shaped to avoid synechiae in this area and facilitate the opening of the frontal recess. Examination of the cells in the frontal recess was then carried out in detail. The agger nasi cell and the frontal cells were observed easily, but the cells on the frontal sinus ostia in the grooves of meatus could not be seen clearly because of the narrow cleft between the uncinate process and the middle turbinate. The cleft of some patients was found to be so close that the uncinate needed to be pushed laterally with an elevator in order to visualize the structures in the grooves of meatus. Afterwards, we were able to use the frontal seeker to explore the ostia in a stepwise manner from posterior to anterior and from medial to lateral. The frontal sinus ostium was found if there was a deep drop in the cavity. It is important to note that the frontal cells or intersinus cells in high locations can appear similar; therefore we employed preoperative CT (Fig. 2) to distinguish this cell. Because the basal lamella of the ethmoid bulla was kept intact, all of the operations were carried out in front of the bulla. We ensured against penetrating into the anterior skull base or the lamina papyracea by giving careful attention to the direction and the power of the seeker. If the ostia were unable to be located after exploration, then the mini-trephine was considered for use.

Finally, the ostia of the frontal sinus were opened. If the ostia of the frontal sinus were identified, it indicated the basal lamella of the uncinate process had been either mostly or completely resected. The ostia of the frontal sinus and the frontal recess were 
then both opened. The use of the seeker or forceps to fracture the bony wall in a forward and downward direction allowed for tracing of the identified ostia. The basal lamella of the uncinate process, the agger nasi cells, and the frontal cells were aimed to be resected completely, which resulted in enlargement of the frontal recess. The posterior membrane needed to be protected so that the round damage to the ostia would not occur, and consequent scarring could be avoided after surgery. After opening the ostia of the frontal sinus, any other necessary sinus or nasal cavity surgery was performed according to the extension or the characteristics of the disease.

Routine postoperative management was carried out following all surgeries. In the frontal recess area, especially the dome of the connection of the anterior middle turbinate and the lateral wall of the nasal cavity, absorbable stanching satin S-100 (Institute of Biologic Material, Beijing, China) covered with Aureomycin (Baijingyu Pharmaceutic, Nanjing, China) ointment was used. In the other cavity, Merocel (Medtronic-Xomed) packing was used according to the extension and hemorrhage condition after surgery. The packing material was cleaned after 24 to 48 hours, and the absorbable material was cleaned after 5 days. A debridement was performed once every 1 to 2 weeks after surgery and then repeated every 2 to 4 weeks, depending on the condition of edema or inflammation in the surgical cavity, until it was epithelized. Nasal lavage with physiologic saline was given for 3 to 6 months, and Fluticasone Propionate Nasal Spray (Glaxo Wellcome Ltd, UK Limited, Spain) or Budesonide Nasal Spray (AstraZeneca PLC, Sodertije, Sweden) was applied until epithelization occurred.

The patients were evaluated for curative effect at the last follow-up evaluation. The specific symptoms of sinusitis that were assessed included overall level of improvement, nasal congestion, facial pain, nasal discharge, headache, smell disorder, and continued use of medications (decongestants, steroids, and antibiotics). The patients were asked to estimate their level of improvement (e.g., significant improvement, mild improvement, no change, or worsening of symptoms) with respect to individual symptoms and overall improvement compared with before their frontal sinus surgery. The ostia patency of the frontal sinus evaluation was graded for the following: completely open, partially narrowed (cannot pass a 4-mm suction tip), or obstructed entirely (no ostia seen).

\section{Results}

The present study was conducted in accordance with international guidelines and approved by the Ethics Committee of Jinling Hospital. In total, 329 $(88.9 \%)$ patients underwent frontal sinus surgery via the route anterior to the ethmoid bulla. Additionally, $27(7.3 \%)$ patients underwent frontal sinus surgery with the help of mini-trephination, $13(3.5 \%)$ patients underwent Draf II procedure, and $1(0.3 \%)$ patient had applied MELP.

There were few complications reported from endoscopic frontal sinus surgery via the route anterior to the ethmoid bulla. Only 2 of the 329 patients reported mild complications with eyelid ecchymosis. There was one anterior ethmoid artery hemorrhage that occurred during the endoscopic frontal sinus surgery with the help of the minitrephination because this particular patient had a narrow frontal recess and variable anterior ethmoid artery. Therefore, when performing the anterior ethmoidectomy procedure, the anterior ethmoid artery was damaged and bled; anterior ethmoid artery ligation was then performed to control the bleeding. Subsequently, this patient had the frontal sinus ostia opened with the help of mini-trephination. Out of the 13 Draf II procedures performed, 1 patient reported eyelid ecchymosis. There were no complications reported in the one MELP procedure (Table 1).

A total of 319 patients (86.2\%) were reported cured, improvement was noted in 36 cases $(9.7 \%)$, and no improvement or worsening of symptoms occurred in 15 patients (4.1\%). At the last follow-up, the ratio of patent ostia of frontal sinus was completely open in 258 patients (69.7\%), partially narrowed in 58 patients (15.7\%), entirely obstructed in 53 patients $(14.3 \%)$, and 1 patient $(0.3 \%)$ needed revision surgery. The last case subsequently underwent the Draf II procedure with the help of minitrephination; after an 18-month follow-up, the ostia were found to be well open. We divided the patients into 2 groups: group A received the endoscopic frontal sinus surgery via the route anterior to the ethmoid bulla procedure, and group B used other procedures. The curative effect and the ratio of frontal sinus patency showed no statistical significance between these two groups (Table 2).

\section{Discussion}

A rhinologist must pursue a safe and convenient operation on the frontal sinus. ${ }^{3}$ Therefore, establish- 
Table 1 The complication ratio of the patient

\begin{tabular}{|c|c|c|c|c|c|c|}
\hline & N (\%) & LPI (\%) & LI (\%) & $\mathrm{EE}(\%)$ & CSF leak (\%) & $\mathrm{AB}(\%)$ \\
\hline FAEB & $329(88.9)$ & $0(0.0)$ & $0(0.0)$ & $2(0.6)$ & $0(0.0)$ & $0(0.0)$ \\
\hline EFMT & $27(7.3)$ & $0(0.0)$ & $0(0.0)$ & $0(0.0)$ & $0(0.0)$ & $1(3.7)$ \\
\hline Draf II & $13(3.5)$ & $0(0.0)$ & $0(0.0)$ & $1(7.7)$ & $0(0.0)$ & $0(0.0)$ \\
\hline MELP & $1(0.3)$ & $0(0.0)$ & $0(0.0)$ & $0(0.0)$ & $0(0.0)$ & $0(0.0)$ \\
\hline Total & $370(100)$ & $0(0.0)$ & $0(0.0)$ & $3(0.8)$ & $0(0.0)$ & $1(0.3)$ \\
\hline
\end{tabular}

$\mathrm{AB}$, anterior ethmoid artery bleeding; EE, eyelid ecchymosis; EFMT, endoscopic frontal sinus surgery with the help of minitrephination; FAEB, frontal sinus surgery in the route anterior to the ethmoid bulla; LI, lacrimal injury; LPI, lamella papyrace injury; MELP, modified endoscopic Lothrop procedure.

ing the proper anatomic marks and proper approach are vital elements of a successful frontal sinus surgery. The traditional process of frontal sinus surgery starts with complete anterior ethmoidectomy, which leads to the frontal sinus according to Messkerlinger and Wigand. ${ }^{4}$ When the anterior ethmoidectomy is performed, a wide view of the anterior ethmoid or the frontal recess is obtained, but the landmarks are damaged and the frontal sinus is opened with significant risks, including blindness.

Louri ${ }^{5}$ first introduced the concept of intact bulla sinusotomy. It has been adapted for the resection of the more anterior ethmoid cells, including the frontal sinus, uncinate process cells, maxillary sinus, and ethmoid infundibulum. Landsberg et al ${ }^{4}$ developed this concept, which isolated frontal sinusitis by using a targeted endoscopic approach. The spirit of this technique is in accordance with microdamage or minimally invasive surgery. We expanded this approach by first opening the frontal sinus in front of the ethmoid bulla and ensuring that the lamella of the ethmoid bulla was complete in most patients.

The ethmoid bulla is a relatively constant anatomic landmark in the middle meatus, although it can have some variation. Its variation is less than the other surrounding structures such as the uncinate process and frontal recess cells. The lamella of the ethmoid bulla is the structure on the anterior wall of the ethmoid bulla and is attached to the anterior skull base, exactly at the junction of the anterior wall and the horizontal wall. Landsberg and Friedman ${ }^{6}$ identified the frontal "beak" and the junction between the anterior base of the skull and the posterior table of the frontal sinus as the frontal ostium. At this plane, the frontal ostium anteriorposterior mean diameter was $7.22 \mathrm{~mm}$, and the transverse mean diameter was $8.92 \mathrm{~mm}$. Therefore, we should only clean the frontal recess cells in front of the ethmoid bulla. The frontal sinus can then be opened, and the ostia do not need to be enlarged.

If the ethmoid bulla is kept intact, and the ostia of the frontal sinus in the anterosuperior direction in front of the lamella of the ethmoid bulla can be accessed, the angle of the operation makes it possible to avoid damage to the anterior skull base in most cases. In most literature, ${ }^{1,4,5}$ the anterior ethmoid artery lies 2 to $3 \mathrm{~mm}$ posterior to the lamella of the ethmoid bulla. Therefore, if the lamella of the ethmoid bulla is left intact, injury to the anterior ethmoid artery can be avoided during surgery. Because of anatomic characteristics, we believe that the approach described here is safer than the traditional approach. In our research, there have been no cerebrospinal fluid (CSF) leaks, lamella papyrace injuries, or lacrimal injury complications from the approach via the route anterior to the ethmoid bulla.

The lamella of the uncinate process is most anterior to the lamella of the ethmoid bone. Its tip lies sagittal and has a close relationship with the agger nasi cell, middle turbinate, lamina papyracea, and anterior skull base. Using coronary CT, Landsberg $^{6}$ divided the attachment of the uncinate process

Table 2 The comparison of group A and group B

\begin{tabular}{|c|c|c|c|c|c|c|c|}
\hline \multirow[b]{2}{*}{ Group } & \multirow[b]{2}{*}{$\mathrm{N}(\%)$} & \multicolumn{3}{|c|}{ Curative effect, n (\%) } & \multicolumn{3}{|c|}{ Frontal sinus patency (\%) } \\
\hline & & Cured & Improvement & Inefficacy & Open & Narrow & None \\
\hline A & 329 & $284(86.3)$ & $32(9.7)$ & $13(4.0)$ & $229(69.6)$ & $52(15.8)$ & $48(14.6)$ \\
\hline B & 41 & $35(85.4)$ & $4(9.8)$ & $2(4.9)$ & $29(70.7)$ & $6(14.6)$ & $5(12.2)$ \\
\hline Mann-Whitney test & & 6674 & & & 5820 & & \\
\hline$P$ & & 0.885 & & & 0.386 & & \\
\hline
\end{tabular}



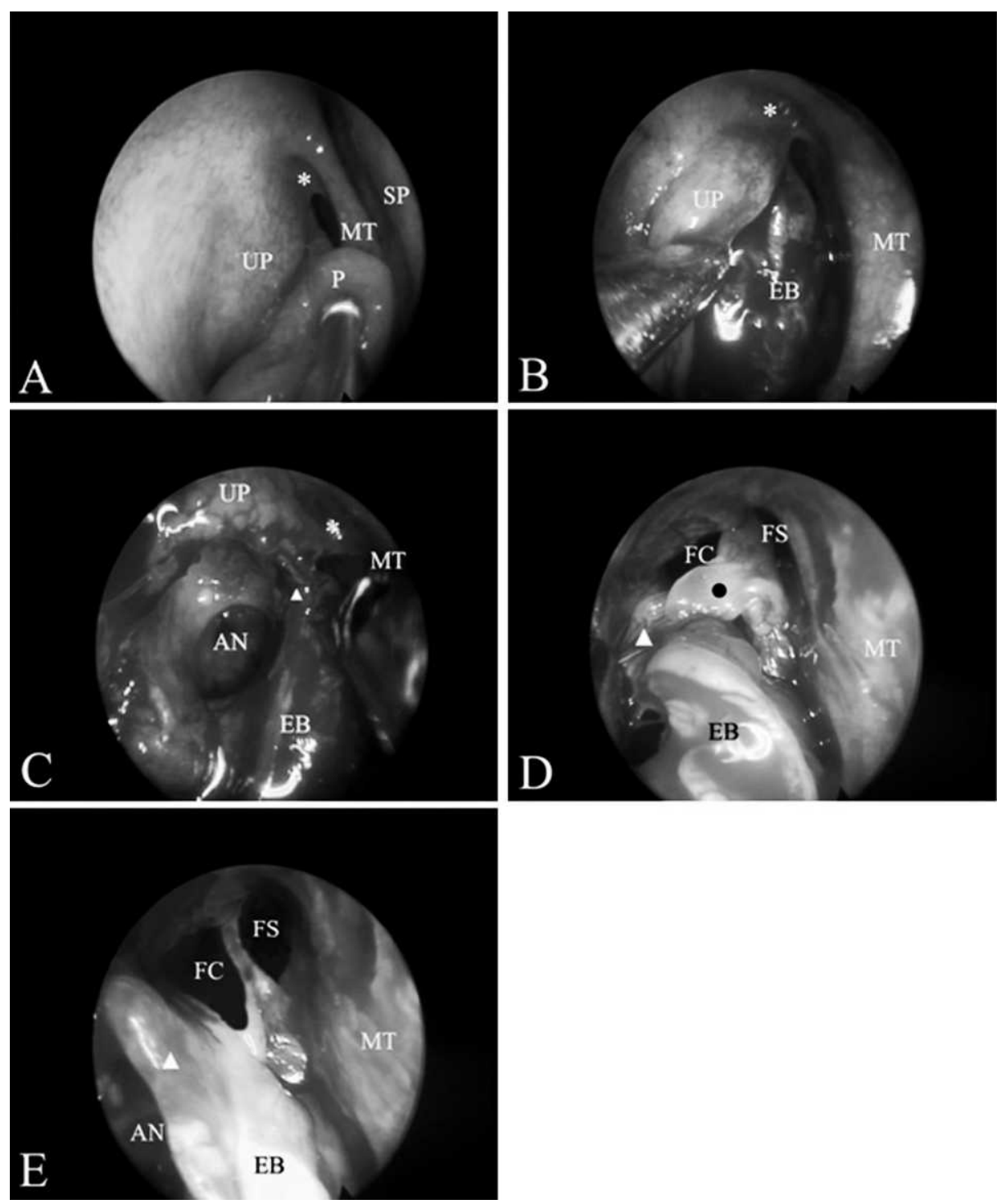

Fig. 1 Surgical technique. (A) Examination before operation. Emergence of a polyp from the middle meatus. The uncinate process and its connection to the middle turbinate is visible (asterisk). SP, nasal septum; MT, middle turbinate; UP, uncinate process; P, polyps. (B) Resection of the polyps, exposure of the ethmoid bulla. EB, ethmoid bulla. (C) The inferior portion of the uncinate process is removed; an agger nasi cell and the relationship of the uncinate process and ethmoid bulla can be seen (little triangle). AN, agger nasi cell. (D) The ostia of frontal sinus has been visible; the polyps (little dot) obstruct the ostia. The connection of the uncinate process and ethmoid bulla was pushed laterally, and the frontal cell can be seen. FC, frontal cell; FS, frontal sinus ostia. (E) The ostia of the frontal sinus has been opened. In the process of the frontal sinus ostia being opened, the ethmoid bulla was kept intact.

into 6 pattern types. Zhang et $a l^{7}$ found that the anterior part of the lamella of the uncinate process has a connection with the attachment of the middle turbinate and the maxillary process, and it consists of the upper, inner, and medial wall of the agger nasi cell. The posterior part of the lamella of the uncinate process' attachment has the same pattern types that Landsberg found. Kim $e t a l^{8}$ and Yoon $e t ~ a l^{9}$ defined the tips of the uncinate process; the supra-infundib- ular plate has the connection with the agger nasi cell and the ethmoid bulla in the sagittal plane, and they believe it is the key to accurately opening the frontal sinus. Some literature from Europe defined the connection of the uncinate process and the ethmoid bulla to the anterior corna. ${ }^{10}$ Therefore, the approach anterior to the ethmoid bulla should be performed while paying close attention to identifying and 

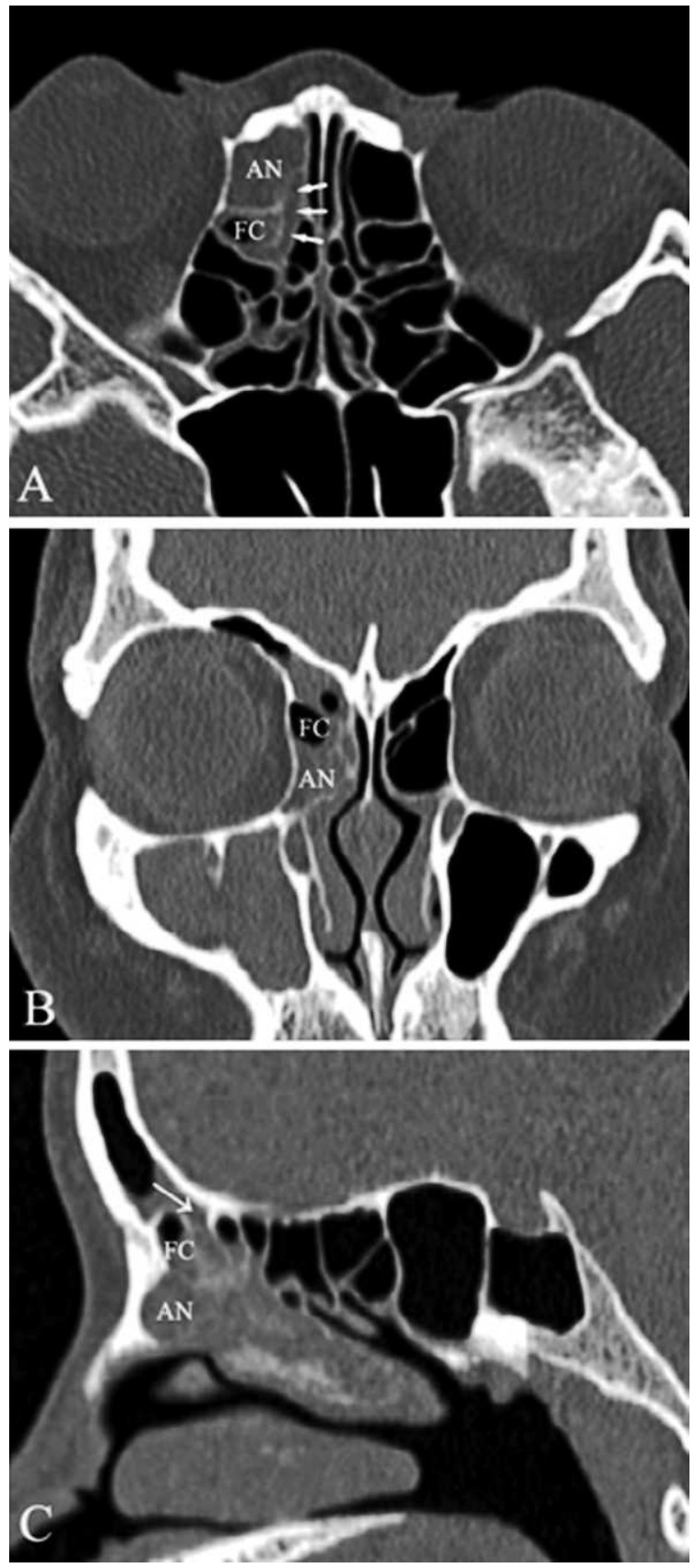

Fig. 2 Preoperative CT sections corresponding to the Figure 1 case. (A) Axial section: a large agger nasi cell and a frontal cell behind it; the frontal sinus outflow (arrow) was medial to these 2 cells. (B) Coronal section: frontal cell above the agger nasi cell. (C) Sagittal section: frontal cell above the agger nasi cell, and the frontal sinus outflow (arrow) was posterior to these 2 cells.

dealing with the lamella of the uncinate process and keeping the ethmoid bulla intact.

There are many cells in the frontal recess. Agger nasi cells and frontal cells are the group of cells at the anterior part of the frontal recess. ${ }^{11}$ Over $90 \%$ of people have the agger nasi cell, so it is important to entirely resect it to assist in opening the frontal sinus. Wormald ${ }^{12}$ completely resected the anterior wall of the agger nasi cell, which made it possible to view the frontal sinus under a zero-degree endoscope. Frontal recess cells, which are the cells above the agger nasi cell, can be divided into 4 types $^{13}$ and make up about $35 \%$ of the cells. They are also in the anterior group of cells in the frontal recess. When they are present, they should be resected upward to ensure the outflow of the frontal sinus.

Interfrontal sinus septal cells are medial, located in the septum of the frontal sinus, and have a prevalence of $12.4 \%$ to $14 \% .{ }^{11,14}$ The posterior groups of the cells in the ethmoid bulla are supraorbital ethmoid cells, suprabullar cells, and frontal bullar cells. They do not disturb the approach via the route anterior to the ethmoid bulla. Therefore, we can say that this procedure can be applied in most cases.

In our research, 329 patients underwent this procedure. However, in some instances, it was not appropriate to pursue an anterior approach to the ethmoid bulla. These situations included bone hyperplasia in the frontal recess, a small anteriorposterior diameter of frontal ostium or the hypogenesis of inner nasal ridge, etc. In these cases, we selected the mini-trephine, Draf II procedure, or the MELP.

Surgeons should carefully prepare the equipment and instruments according to the endoscopic examination and perform CT and other necessary examinations preoperatively. If it is difficult to keep the ethmoid bulla, do not persist. Mini-trephine is a very good option as it can help the surgeon locate the ostia of the frontal sinus easily and safely and will not make an obvious scar on the face. Therefore, if the surgeon does not have enough confidence in being able to open the ostia or if the surgical condition is complicated, do not hesitate to use the mini-trephine.

During the follow-up evaluation, the patency of the ostium of the frontal sinus could not be confirmed in $14.3 \%$ of cases. In most cases, however, the patients reported feeling better than they had preoperatively. Although the results of the images under endoscope were not satisfactory to the doctor, most patients were satisfied with the results. Therefore, we advised these patients to have a long nasal flush and to use nasal topical corticosteroids. 


\section{Acknowledgments}

This study has been supported by a grant from the Natural Science Fund of Jiangsu Province, China (BK2006134).

\section{References}

1. Friedman Vidyasagar R, Landsberg R. Frontal sinus surgery 2004: Update of clinical anatomy and surgical techniques. Oper Tech Otolaryngol Head Neck Surg 2004;15(1):23-31

2. Kim SS, Lee JG, Kim KS, Kim HU, Chung IH, Yoon JH. Computed tomographic and anatomical analysis of the basal lamellas in the ethmoid sinus. Laryngoscope 2001;111(3):424429

3. Jacobs JB. 100 years of frontal sinus surgery. Laryngoscope 1997; 107(11, pt 2):1-36

4. Landsberg R, Segev Y, Friedman M, Fliss DM, Derowe A. A targeted endoscopic approach to chronic isolated frontal sinusitis. Otolaryngol Head Neck Surg 2006;134(1):28-32

5. Louri MC. Endoscopic frontal recess and frontal sinus ostium dissection. Laryngoscope 1993;113:455-458

6. Landsberg R, Friedman M. A computer-assisted anatomical study of the nasofrontal region. Laryngoscope 2001;111(12): 2125-2130
7. Zhang L, Han D, Ge W, Xie J, Zhou B, Fan E et al. Anatomical and computed tomographic analysis of the interaction between the uncinate process and the agger nasi cell. Acta Otolaryngol 2006;126(8):845-852

8. Kim KS, Kim HU, Chung IH, Lee JG, Park IY, Yoon JH. Surgical anatomy of the nasofrontal duct: anatomical and computed tomographic analysis. Laryngoscope 2001;111(4, pt 1):603-608

9. Yoon JH, Moon HJ, Kim CH, Hong SS, Kang SS, Kim K. Endoscopic frontal sinusotomy using the suprainfundibular plate as a key landmark. Laryngoscope 2002;112(9):1703-1707

10. Agrifoglio A, Terrier G, Duvoisin B. Anatomic and endoscopic studies of the anterior ethmoid. Ann Otolaryngol Chir Cervicofac 1990;107(4):249-258

11. Lee WT, Kuhn FA, Citardi MJ. 3D computed tomographic analysis of frontal recess anatomy in patients without frontal sinusitis. Otolaryngol Head Neck Surg 2004;131(3):164-173

12. Wormald PJ. The agger nasi cell: the key to understanding the anatomy of the frontal recess. Otolaryngol Head Neck Surg 2003; 129(5):497-507

13. Kuhn FA. Chronic frontal sinusitis: the endoscopic frontal recess approach. Oper Tech Otolaryngol Head Neck Surg 1996; 17(3):222-229

14. Zhang L, Han D, Ge W, Tao J, Wang X, Li Y et al. Computed tomographic and endoscopic analysis of supraorbital ethmoid cells. Otolaryngol Head Neck Surg 2007;137(4):562-568 